\title{
Prevalência de fatores de risco para doenças cardiovasculares na população Guaraní-Mbyá do Estado do Rio de Janeiro
}

\author{
Prevalence of risk factors for cardiovascular \\ disease in the Guaraní-Mbyá population \\ of the State of Rio de Janeiro
}

Andrey M. Cardoso 1

Inês E. Mattos 1

Rosalina J. Koifman 1

\footnotetext{
1 Departamento de Epidemiologia e Métodos Quantitativos em Saúde, Escola Nacional de Saúde Pública, Fundação Oswaldo Cruz. Rua Leopoldo Bulhões 1480, Rio de Janeiro, $R J$ 21041-210, Brasil.
}

\begin{abstract}
Social change has been involved in the unequal distribution patterns of chronic diseases in several populations. Among Indian communities experiencing life pattern changes, international studies have reported increased prevalence of hypertension and other cardiovascular risk factors. Such increased prevalence was ascertained in a survey conducted in 1999 in selected Guarani-Mbyá communities (Sapukai, Paraty-Mirim, and Araponga) in the State of Rio de Janeiro, Brazil. A population census was carried out and interviews and clinical and biochemical evaluations were conducted with 80 men and 71 women. Observed prevalence of selected risk factors in the overall sample, including men and women, was as follows for the three communities: hypertension (4.8\%, 2.6\%, 7.4\%); overweight (26.7\%, 19.5\%, 34.8\%); total cholesterol levels (2.8\%, 2.7\%, 2.9\%), and increased triglyceride levels (12.6\%, 9.5\%, 15.9\%). All prevalence rates were higher among women and at older ages. The results suggest that the Guarani communities have a moderate risk of chronic diseases and that measures to reduce these risk factors should be adopted.
\end{abstract}

Key words Anthropometry; Chronic Diseases; Hypertension; Guaraní-Mbyá; South American Indians

Resumo A distribuição desigual das doenças crônico-degenerativas tem sido atribuída aos diferentes graus de transformação social em diversas populações. Estudos internacionais em populações indígenas submetidas a mudanças em seus estilos de vida têm mostrado prevalências elevadas de hipertensão arterial e de outros fatores de risco cardiovasculares. Foi feito um estudo de prevalência desses fatores de risco na população adulta das aldeias indígenas Sapukai, Paraty-Mirim e Araponga no Rio de Janeiro. Após recenseamento, a população teve os dados coletados através de entrevista e de avaliações clínicas e bioquímicas. O universo estudado foi de 80 homens e 71 mulheres. As prevalências em toda a amostra foram respectivamente para: hipertensão arterial (4,8\%, 2,6\% e 7,4\%); sobrepeso $(26,7 \%, 19,5 \%$ e 34,8\%) e obesidade $(4,8 \%, 3,9 \%$ e 5,8\%); alterações lipídicas do colesterol total (2,8\%, 2,7\% e 2,9\%) e dos triglicerídios (12,6\%, 9,5\% e 15,9\%). Todas as prevalências foram superiores no sexo feminino e maiores nas idades mais avançadas. Os resultados sugerem que a população avaliada encontra-se sob risco intermediário para as doenças crônicas, mostrando que devem ser empreendidos esforços para controlar os fatores de risco.

Palavras-chave Antropometria; Doença Crônica; Hipertensão; Guaraní-Mbyá; Índios SulAmericanos 


\section{Introdução}

Alguns estudos populacionais vêm chamando a atenção para a maior suscetibilidade às doenças crônicas em grupos submetidos à modernização de seu estilo de vida (Fleming-Moran \& Coimbra Jr., 1990; Fleming-Moran et al., 1991; Young, 1988; Welty et al., 1995). Mudanças na dieta, estresse psicológico, sedentarismo, obesidade, hereditariedade e mudanças sócio-econômicas têm sido considerados fatores contribuintes para essa situação. Tal fato pode ser exemplificado com a população indígena norte-americana, em que as doenças cardiovasculares se tornaram uma das principais causas de mortalidade (Mendlein et al., 1997; Percy et al., 1997; Rhoades et al., 1987; Welty et al., 1995).

Estudos com populações indígenas têm demonstrado a ocorrência de aumento das doenças cardiovasculares, de diabetes e de transtornos mentais em razão do processo de crise de identidade cultural e de mudanças de hábitos, ressaltando-se os alimentares. Embora nessas populações venha sendo cada vez mais freqüente a detecção de mudanças comportamentais e de casos de doenças crônicas após o contato com a população não-indígena, é ainda comum a coexistência de padrão epidemiológico composto por doenças infecto-contagiosas e carenciais (Bloch et al., 1993; Coimbra Jr. \& Santos, 1991; Hern, 1991; Leite, 1998; Santos \& Coimbra Jr., 1994).

Em populações autóctones e não-autóctones tem sido demonstrado que a pressão arterial apresenta comportamento variado em função do grau de ocidentalização do estilo de vida (Achutti \& Medeiros, 1985; Bloch et al., 1993; Carvalho et al., 1989; Dustan, 1991; FlemingMoran et al., 1991; Hern, 1991; INTERSALT, 1988; Lolio et al., 1993), assim como outros fatores de risco para doenças crônico-degenerativas, tais como obesidade e dislipidemias.

Os grupos indígenas Guaraní-Mbyá que vivem no litoral do Estado do Rio de Janeiro têm experimentado profundo contato interétnico, ao passo que a destruição dos ecossistemas vem interferindo nos aspectos mais diversos de sua vida (Litaiff, 1996). Apesar do contato permanente, a situação de saúde dessas comunidades - em especial, da população adulta - ainda é pouco conhecida, e a garantia da "assistência” à saúde e da adequação dos serviços às características sócio-culturais e necessidades reais desses grupos depende de melhor conhecimento da realidade. Esse cenário levou à realização de estudo com o objetivo de avaliar a prevalência dos fatores de risco cardiovasculares na população adulta Guaraní-Mbyá do litoral do Rio de Janeiro.

\section{População e métodos}

Através de censo populacional foram identificados todos os indígenas moradores atuais das três aldeias Guaraní-Mbyá do Rio de Janeiro. Foram elegíveis para o estudo 193 indígenas de ambos os sexos, com idade igual ou superior a 15 anos, segundo os registros de nascimento da FUNAI ou o documento de identidade. Na ausência desses documentos, foi considerada a idade aproximada - levando-se em conta a referida pelo indivíduo - e confrontada com a idade aparente. Após a obtenção do consentimento informado, os indivíduos foram submetidos a exame físico, a medidas antropométricas e a coleta de material para exames laboratoriais.

$O$ peso foi aferido em gramas através de balança portátil digital calibrada, e a altura, em centímetros, através de fita métrica invertida e posicionada a $50 \mathrm{~cm}$ do piso, em parede plana sem rodapé e esquadro de madeira revestido de fórmica. O Índice de Massa Corporal (IMC) foi calculado pela fórmula [peso $(\mathrm{kg}) /$ altura $^{2}$ $\left.\left(\mathrm{m}^{2}\right)\right]$. Os critérios de classificação do IMC foram os preconizados pela Organização Mundial da Saúde - OMS (WHO, 1995), que adota como graus de baixo peso I, II e III respectivamente, $17,0 \leq \mathrm{IMC}<18,5,16,0 \leq \mathrm{IMC}<17,0 \mathrm{e}$ IMC $<16,0$; como peso adequado 18,5 $\leq$ IMC $<$ 25,0 , enquanto avalia como sobrepeso os graus I, II e III, respectivamente, 25,0 $\leq$ IMC $<30,0$, $30,0 \leq$ IMC $<40,0$ e IMC $\geq 40,0$.

As circunferências da cintura e do quadril foram medidas por intermédio de fita métrica de material sintético não extensível, graduada em milímetros (WHO, 1995). Valores de Razão cintura-quadril (RCQ) superiores a 0,95 para homens, a 0,80 para mulheres sem sobrepeso e a 0,85 para mulheres com sobrepeso foram considerados de risco (Pereira et al., 1999).

A pressão arterial (PA) foi aferida através de esfigmomanômetro zero-randômico Hawksley, com manguito padrão para adulto (23 por $12 \mathrm{~mm}$ ) devidamente calibrado e estetoscópio Littmann Cardiology. As medidas obtidas com os instrumentos utilizados foram corrigidas segundo o perímetro braquial do indivíduo examinado (Fuchs, 1996). Foram efetuadas duas medidas de PA no domicílio e duas medidas no ambulatório. A média de cada grupo de duas medidas foi considerada o valor observado no indivíduo. Os indivíduos foram classificados como portadores de hipertensão arterial segundo dois critérios: o do Joint National Committee (JNC, 1988) - que considera hipertensão valores iguais ou superiores a 140 x $90 \mathrm{mmHg}-$ e o da OMS (WHO, 1978), que considera valores iguais ou superiores a $160 \times 95 \mathrm{mmHg}$. 
Em relação às dislipidemias, foram adotados os critérios de classificação apresentados pelo relatório do segundo painel de especialistas do National Cholesterol Education Program (Tabela 1; NCEP, 1993). Todos os lipídios foram classificados, tomando-se como risco ainda os valores limítrofes. Em relação ao colesterolHDL, o ponto de corte das mulheres acima de 50 anos foi igual ao masculino $(35 \mathrm{mg} / \mathrm{dl})$.

Do total de indígenas selecionados, 16 indivíduos $(8,3 \%)$ recusaram-se a participar do estudo e quatro $(2,1 \%)$ mudaram-se antes de serem avaliados. O grupo de 16 gestantes $(18,4 \%)$ foi excluído da análise nesta publicação. Dentre os indígenas avaliados, 6 (3,5\%) foram entrevistados e submetidos à coleta de sangue, mas não quiseram ser examinados.

A análise dos dados consistiu na exploração descritiva dos parâmetros populacionais e nos cálculos das prevalências de valores anormais das variáveis pressão arterial (PA), Colesterol total, HDL, LDL, triglicerídios e das variáveis antropométricas peso, estatura, IMC e RCQ. Foi avaliado também o grau de correlação entre as variáveis por intermédio do coeficiente de correlação de Pearson, em análises bivariadas, testando-se a significância estatística através do teste t bi-caudal.

Optou-se pela análise em diferentes estratos de idade, definidos pelos intervalos de classe 15 a 29 anos, 30 a 49 anos e 50 anos e mais, para descrever as distribuições das variáveis estudadas.

\section{Resultados}

A população de estudo é constituída por 80 homens $(53,0 \%)$ e 71 mulheres $(47,0 \%)$, com 76 indivíduos $(50,3 \%)$ concentrados na faixa etária de 15 a 29 anos. A distribuição da população segundo sexo e faixa etária encontra-se na Tabela 2.

\section{Variáveis antropométricas}

\section{- Peso e estatura}

As médias de peso e estatura e os respectivos desvios padrão encontram-se na Tabela 3 .

\section{- Índice de massa corporal}

O grupo feminino apresentou média de IMC semelhante à do masculino. Observou-se apenas baixo peso (BP) grau I. A prevalência global de BP foi $4,1 \%$, sendo respectivamente de $3.9 \%$ e $4,3 \%$ nos sexos masculino e feminino. No gru-
Tabela 1

\begin{tabular}{|c|c|c|}
\hline Tipo & Classificação & Valores \\
\hline \multirow[t]{3}{*}{ Colesterol total } & Desejável & até $200 \mathrm{mg} / \mathrm{dl}$ \\
\hline & Limítrofe (risco) & de $200 \mathrm{mg} / \mathrm{dL}$ até $240 \mathrm{mg} / \mathrm{dL}$ \\
\hline & Elevado & acima de $240 \mathrm{mg} / \mathrm{dL}$ \\
\hline \multirow[t]{2}{*}{ HDL-colesterol } & Desejável & Homens e mulheres $>50$ : acima de $35 \mathrm{mg} / \mathrm{dL}$ \\
\hline & & Mulheres: acima de $45 \mathrm{mg} / \mathrm{dL}$ \\
\hline \multirow[t]{3}{*}{ LDL-colesterol } & Desejável & até $130 \mathrm{mg} / \mathrm{dL}$ \\
\hline & Limítrofe (risco) & de $130 \mathrm{mg} / \mathrm{dL}$ até $160 \mathrm{mg} / \mathrm{dL}$ \\
\hline & Elevado & acima de $160 \mathrm{mg} / \mathrm{dL}$ \\
\hline \multirow[t]{3}{*}{ Triglicerídios } & Desejável & até $200 \mathrm{mg} / \mathrm{dl}$ \\
\hline & Limítrofe (risco) & de $200 \mathrm{mg} / \mathrm{dL}$ até $499 \mathrm{mg} / \mathrm{dL}$ \\
\hline & Elevado & acima de $499 \mathrm{mg} / \mathrm{dL}$ \\
\hline
\end{tabular}

Fonte: NCEP (1993).

Tabela 2

Distribuição da população indígena Guaraní-Mbyá segundo sexo e faixa etária.

\begin{tabular}{lrrrrrrr}
\hline \multirow{2}{*}{ Faixa Etária } & \multicolumn{2}{c}{ Masculino } & \multicolumn{2}{c}{ Feminino } & \multicolumn{2}{c}{ Global } \\
& $n$ & $\%$ & \multicolumn{2}{c}{$\mathrm{n}$} & $\%$ & $\mathrm{n}$ & $\%$ \\
\hline 15 a 29 & 43 & 53.7 & 33 & 46.5 & 76 & 50.3 \\
30 a 49 & 19 & 23.8 & 13 & 18.3 & 32 & 21.2 \\
50 e mais & 18 & 22.5 & 25 & 35.2 & 43 & 28.5 \\
Global & 80 & 100.0 & 71 & 100.0 & 151 & 100.0 \\
\hline
\end{tabular}

po feminino houve aumento do BP na faixa etária mais velha, enquanto no grupo masculino, só se detectou BP nos mais jovens (Tabela 4).

Não foram observados valores compatíveis com sobrepeso (SP) grau III. A prevalência global de SP foi $26,7 \%$, e os grupos masculino e feminino apresentaram respectivamente as prevalências de $19,5 \%$ e $34,8 \%$.

A prevalência global de SP I foi de 21,9\%. No sexo masculino, esse valor correspondeu a $15,6 \%$ e, no feminino, a $29,0 \%$.

A prevalência global de SP II (obesidade) foi de $4,8 \%$ e de, respectivamente, $3,9 \%$ e $5,8 \%$ nos sexos masculino e feminino. No grupo feminino, não se observou obesidade na faixa de idade intermediária, enquanto no grupo masculino registrou-se aumento progressivo até os 49 anos e ausência de obesidade acima dessa idade (Tabela 4). 
Tabela 3

Média e desvio padrão de variáveis selecionadas na população do estudo.

\begin{tabular}{|c|c|c|c|}
\hline Variável & Masculino & Feminino & Global \\
\hline \multicolumn{4}{|l|}{ Idade } \\
\hline Número & 80 & 71 & 151 \\
\hline Média & 36,2 & 41,2 & 38,6 \\
\hline Desvio Padrão & 20,8 & 23,3 & 146 \\
\hline \multicolumn{4}{|l|}{ Peso } \\
\hline Número & 77 & 69 & 54,1 \\
\hline Média & 57,4 & 50,4 & 8,7 \\
\hline Desvio Padrão & 7,2 & 8,7 & 22,1 \\
\hline \multicolumn{4}{|l|}{ Estatura } \\
\hline Número & 77 & 69 & 0,08 \\
\hline Média & 1,58 & 1,45 & 146 \\
\hline Desvio Padrão & 0,05 & 0,06 & 1,52 \\
\hline \multicolumn{4}{|l|}{ IMC } \\
\hline Número & 77 & 69 & 146 \\
\hline Média & 23,2 & 23,9 & 23,5 \\
\hline Desvio Padrão & 2,9 & 3,7 & 3,3 \\
\hline \multicolumn{4}{|l|}{$\mathrm{RCQ}$} \\
\hline Número & 77 & 69 & 146 \\
\hline Média & 0,87 & 0,85 & 0,05 \\
\hline Desvio Padrão & 0,04 & 0,05 & 0,86 \\
\hline \multicolumn{4}{|l|}{ PAS } \\
\hline Número & 77 & 68 & 145 \\
\hline Média & 109,6 & 108,9 & 109,3 \\
\hline Desvio Padrão & 10,4 & 16,4 & 13,6 \\
\hline \multicolumn{4}{|l|}{ PAD } \\
\hline Número & 78 & 68 & 145 \\
\hline Média & 69,8 & 69,5 & 69,7 \\
\hline Desvio Padrão & 9,2 & 10,4 & 9,8 \\
\hline \multicolumn{4}{|l|}{ Colesterol } \\
\hline Número & 74 & 69 & 143 \\
\hline Média & 139,4 & 148,5 & 143,8 \\
\hline Desvio Padrão & 28,1 & 29,1 & 28,8 \\
\hline \multicolumn{4}{|l|}{ HDL } \\
\hline Número & 74 & 69 & 143 \\
\hline Média & 34,8 & 38,0 & 36,3 \\
\hline Desvio Padrão & 10,6 & 9,3 & 10,1 \\
\hline \multicolumn{4}{|l|}{ LDL } \\
\hline Número & 72 & 68 & 141 \\
\hline Média & 82,7 & 86,4 & 84,1 \\
\hline Desvio Padrão & 21,4 & 21,6 & 22,1 \\
\hline \multicolumn{4}{|l|}{ Triglicerídios } \\
\hline Número & 74 & 69 & 143 \\
\hline Média & 113,3 & 119,0 & 116,0 \\
\hline Desvio Padrão & 62,5 & 86,5 & 74,8 \\
\hline
\end{tabular}

\section{- Razão cintura-quadril}

A média global de RCQ foi 0,86. A média feminina foi superior ao valor de referência considerado de risco para doenças crônicas nesse grupo.

A análise segundo faixa etária mostrou que, no sexo masculino, a maior média de RCQ ocorreu no grupo de 30 a 49 anos $(0,90)$ e todas as médias foram inferiores ao valor de referência para risco de doenças crônicas no sexo masculino. No grupo feminino, as médias foram discretamente crescentes segundo as faixas etárias $(15-29: 0,83 ; 30-49: 0,86$; 50 e mais: 0,87$)$ e superiores ao valor de risco, em todos os estratos de idade.

A prevalência global de concentração abdominal de gordura foi $44,5 \%$, mostrando-se maior no sexo feminino $(86,9 \%)$ do que no masculino $(6,5 \%)$. Quando utilizado 0,85 como ponto de corte para o grupo feminino com SP (Pereira et al., 1999), a prevalência global passou a ser $39,7 \%$ e a feminina, $75,4 \%$. A análise estratificada por sexo e idade mostrou prevalências de $71,9 \%, 92,3 \%$ e $70,8 \%$ no sexo feminino e $2,4 \%$, $16,7 \%$ e $14,3 \%$ no sexo masculino, respectivamente para as faixas etárias de 15-29, 30-49 e 50 e mais.

\section{Pressão arterial}

As médias globais de pressões arteriais sistólica (PAS) e diastólica (PAD) foram inferiores no ambulatório (PAS: 109,3mmHg; PAD: 69,7mmHg) quando comparadas às médias globais domiciliares (PAS: 110,8mmHg; PAD:71,0mmHg), o mesmo ocorrendo quando a análise foi feita separadamente para os sexos. Optou-se pela utilização das médias ambulatoriais para cálculo da prevalência de hipertensão arterial, supondo que seus valores estejam menos influenciados pelo estresse da avaliação médica.

Dentre os indígenas avaliados, seis indivíduos foram classificados como portadores de hipertensão arterial segundo o critério JNC (1988) e um, por fazer uso regular de medicação anti-hipertensiva, correspondendo a uma prevalência global de $4,8 \%$. A análise por sexo mostrou prevalência de $2,6 \%$ no sexo masculino e de $7,4 \%$ no sexo feminino.

Segundo critério da OMS (WHO, 1978), as prevalências de hipertensão arterial global e para o sexo feminino foram respectivamente de $2,1 \%$ e $4,4 \%$. Segundo esse parâmetro, nenhum indígena do sexo masculino foi classificado como hipertenso.

Em relação às faixas etárias, as prevalências foram maiores na faixa etária de 50 anos e mais 
(JNC: 9,8\%; OMS: 7,3\%), seguida pela faixa etária de 15 a 29 anos (JNC: $4,1 \%$ ) para qualquer dos critérios utilizados. Nenhum indígena de 30 a 49 anos foi classificado como hipertenso e, pelo critério da OMS, somente indígenas com 50 anos e mais foram classificados como tal. $\mathrm{O}$ padrão acima manteve-se em relação aos sexos, com prevalências de $16,4 \%$ e $12,5 \%$, ambas para o grupo feminino de 50 anos e mais, respectivamente pelos critérios do JNC e da OMS. Na faixa etária de 15 a 29 anos, a prevalência pelo critério JNC, ao contrário do observado até o momento, foi superior no grupo masculino $(4,8 \%)$, quando comparado ao feminino $(3,2 \%)$.

\section{Colesterol e triglicerídios}

As média globais de colesterol total, colesterolLDL e triglicerídios foram inferiores aos respectivos valores de referência, enquanto as médias de colesterol-HDL, para os dois sexos se incluem na faixa considerada de risco (Tabela 5). Ao avaliar separadamente os sexos, as médias femininas de colesterol total, HDL e LDL e triglicerídios foram superiores às masculinas.

As prevalências globais e por sexo de elevação de colesterol total (G: 2,8\%, M: 2,7\%, F: 2,9\%) e LDL (G: 2,8\%, M: 2,7\%, F: 2,9\%) foram bastante inferiores às prevalências de elevação de triglicerídios (G: 12,6\%, M: 9,5\%, F: 15,9\%) e, sobretudo, de HDL (G: 62,2\%, M: 58,1\%, F: 66,7\%).

\section{Correlações (Tabela 6)}

\section{- Idade}

A idade apresentou correlações significativas com quase todas as variáveis estudadas. Ressaltam-se as correlações positivas com as PAS e PAD, as correlações inversas com a estatura e o peso (não significativa) e a fraca associação com o IMC.

A correlação inversa entre estatura e idade manteve-se na análise por sexo, evidenciandose estatisticamente significativa no sexo feminino $(-0,295, \mathrm{p}<0,05)$.

\section{- Medidas antropométricas}

O peso apresentou correlação significativa com a maioria das variáveis, sendo as mais fortes com a estatura, com o IMC e com a RCQ. Destaca-se a correlação positiva com a PAD - que desapareceu no sexo masculino $(+0,19, \mathrm{p}>0,05)$ e ficou mais forte no feminino $(+0,30, \mathrm{p}<0,05)$ - e também a significativa elevação da força de associação (inversa) com o HDL $(-0,33, \mathrm{p}<0,01)$, no grupo feminino.

\begin{tabular}{|c|c|c|c|c|}
\hline \multicolumn{5}{|c|}{ Prevalências de IMC segundo sexo e faixa etária. } \\
\hline \multirow{2}{*}{$\begin{array}{l}\text { Sexo e faixa } \\
\text { etária }\end{array}$} & \multicolumn{4}{|c|}{ Prevalência } \\
\hline & Baixo peso & Peso adequado & Sobrepeso I & $\begin{array}{l}\text { Sobrepeso II } \\
\text { (obesidade) }\end{array}$ \\
\hline \multicolumn{5}{|l|}{ Masculino } \\
\hline 15 a 29 & 7,1 & 83,3 & 7,1 & 2,4 \\
\hline 30 a 49 & 0,0 & 50,0 & 38,9 & 11,1 \\
\hline 50 e + & 0,0 & 88,2 & 11,8 & 0,0 \\
\hline \multicolumn{5}{|l|}{ Feminino } \\
\hline 15 a 29 & 3,1 & 71,9 & 18,8 & 6,2 \\
\hline 30 a 49 & 0,0 & 53,8 & 46,2 & 0,0 \\
\hline 50 e + & 8,3 & 50,0 & 33,4 & 8,3 \\
\hline \multicolumn{5}{|l|}{ Global } \\
\hline 15 a 29 & 5,4 & 78,4 & 12,2 & 4,0 \\
\hline 30 a 49 & 0,0 & 51,6 & 41,9 & 6,5 \\
\hline 50 e + & 4,9 & 65,8 & 24,4 & 4,9 \\
\hline
\end{tabular}

A estatura apresentou correlações fracas e inversas com a maioria das variáveis, destacando-se a inversão do sentido da associação entre ela e a PAS $(-0,10, p>0,05)$ no sexo feminino.

O IMC apresentou forte correlação apenas com o peso. Ressalta-se a correlação significativa com a PAD (global) e a inversão do sentido da correlação com a PAS $(+0,08, p>0,05)$ no sexo masculino, embora sem significância estatística. No sexo feminino, destaca-se o aumento da força de associação positiva com as variáveis lipídicas e com a PAD (colesterol: $+0,35$, $\mathrm{p}<$ 0,01; HDL: -0,33, p < 0,01; LDL: +0,31, p < 0,05; triglicerídios: $+0,32, \mathrm{p}<0,01$ e PAD: $+0,35, \mathrm{p}<$ $0,01)$.

Todas as correlações apresentadas pela RCQ foram fracas, destacando-se a correlações positivas com a idade (significativa) e com a estatura (não significativa). Na análise por sexo, a correlação positiva com a estatura se inverteu (M: -0,11 e F: -0,22, p>0,05) e as correlações com as PAS $(+0,20, \mathrm{p}>0,05)$ e PAD $(+0,27, \mathrm{p}<0,01)$ tornaram-se positivas no grupo feminino.

\section{- Pressão arterial}

A PAS somente apresentou forte correlação com a PAD. Destacam-se as correlações inversas com as variáveis lipídicas e antropométricas (IMC: -0,08 e RCQ: -0,07; p > 0,05) no sexo masculino e com a estatura, no sexo feminino, para ambas as pressões arteriais (PAS: $+0,10$ e PAD: $+0,10 ; \mathrm{p}>0,05)$. 
Tabela 5

Médias e desvios-padrão de colesterol total, HDL, LDL e Triglicerídios, segundo sexo e faixa etária.

\begin{tabular}{|c|c|c|c|c|c|c|c|c|c|c|c|c|}
\hline \multirow{2}{*}{$\begin{array}{l}\text { Sexo e } \\
\text { Faixa Etária }\end{array}$} & \multicolumn{3}{|c|}{ Colesterol } & \multicolumn{3}{|c|}{ HDL } & \multicolumn{3}{|c|}{ LDL } & \multicolumn{3}{|c|}{ Triglicerídios } \\
\hline & $n$ & Média & DP & $\mathrm{n}$ & Média & DP & $\mathrm{n}$ & Média & DP & $\mathrm{n}$ & Média & DP \\
\hline \multicolumn{13}{|l|}{ Masculino } \\
\hline $15-29$ & 38 & 127,2 & 20,875 & 38 & 30,7 & 7,360 & 37 & 75,4 & 19,176 & 38 & 104,1 & 57,272 \\
\hline $30-49$ & 18 & 155,6 & 30,849 & 18 & 36,6 & 7,358 & 18 & 92,8 & 25,178 & 18 & 131,9 & 70,856 \\
\hline $50 e+$ & 18 & 149,2 & 27,667 & 18 & 41,6 & 14,869 & 18 & 84,3 & 22,157 & 18 & 114,0 & 63,773 \\
\hline \multicolumn{13}{|l|}{ Feminino } \\
\hline $15-29$ & 32 & 137,6 & 25,650 & 32 & 39,1 & 9,623 & 32 & 79,4 & 23,168 & 32 & 90,9 & 38,007 \\
\hline $30-49$ & 12 & 146,2 & 23,254 & 12 & 39,3 & 6,269 & 12 & 88,2 & 17,383 & 12 & 95,8 & 64,675 \\
\hline 50 e + & 25 & 163,4 & 30,533 & 25 & 36,0 & 10,054 & 24 & 94,8 & 18,591 & 25 & 165,8 & 117,553 \\
\hline \multicolumn{13}{|l|}{ Global } \\
\hline $15-29$ & 70 & 132,0 & 23,595 & 70 & 34,5 & 9,405 & 69 & 77,2 & 21,062 & 70 & 98,1 & 49,511 \\
\hline $30-49$ & 30 & 151,9 & 28,013 & 30 & 37,7 & 6,955 & 30 & 91,0 & 22,172 & 30 & 117,5 & 69,667 \\
\hline $50 e+$ & 43 & 157,4 & 29,882 & 43 & 38,3 & 12,447 & 42 & 90,3 & 20,618 & 43 & 144,1 & 101,045 \\
\hline
\end{tabular}

Tabela 6

Matriz de Correlação de Pearson para os sexos combinados.

\begin{tabular}{|c|c|c|c|c|c|c|c|c|c|c|c|}
\hline Variáveis & Estatura & Colesterol & HDL & Idade & IMC & LDL & Peso & $\mathrm{RCO}$ & Triglicerídios & PAS & PAD \\
\hline Estatura & - & & & & & & & & & & \\
\hline Colesterol & $-0,125$ & - & & & & & & & & & \\
\hline HDL & $-0,094$ & $0,271^{\star \star}$ & - & & & & & & & & \\
\hline Idade & $-0,214^{\star \star}$ & $0,364^{\star \star}$ & $0,202^{\star}$ & - & & & & & & & \\
\hline IMC & $-0,105$ & $0,285^{\star \star}$ & $-0,207^{\star}$ & 0,041 & - & & & & & & \\
\hline LDL & $-0,100$ & $0,856^{\star \star}$ & 0,081 & $0,236^{\star \star}$ & $0,233^{\star \star}$ & - & & & & & \\
\hline Peso & $0,585^{\star \star}$ & $0,197^{\star}$ & $-0,202^{\star}$ & $-0,091$ & $0,670^{\star \star}$ & 0,148 & - & & & & \\
\hline $\mathrm{RCQ}$ & 0,111 & $0,306^{\star *}$ & $-0,012$ & $0,273^{\star *}$ & $0,271^{\star \star}$ & $0,172^{*}$ & $0,347^{\star \star}$ & - & & & \\
\hline Triglicerídios & $-0,027$ & $0,538^{\star \star}$ & $-0,244^{\star \star}$ & $0,238^{\star \star}$ & $0,318^{\star \star}$ & 0,164 & $0,276^{\star \star}$ & $0,366^{\star \star}$ & - & & \\
\hline PAS & 0,038 & 0,070 & 0,005 & $0,194^{\star}$ & 0,116 & $-0,004$ & 0,139 & 0,076 & 0,146 & - & \\
\hline PAD & 0,055 & $0,220^{\star *}$ & $-0,012$ & $0,200^{*}$ & $0,228^{\star \star}$ & $0,170^{*}$ & $0,235^{\star \star}$ & 0,073 & $0,193^{*}$ & $0,680^{\star *}$ & - \\
\hline
\end{tabular}

** $\mathrm{P}$-valor $<0,01$ (teste $\mathrm{t}$ bi-caudal).

* P-valor $<0,05$ (teste t bi-caudal).

\section{Discussão}

Os dados antropométricos mostram que os Guaraní de ambos os sexos são mais altos e mais pesados que os Yanomámi (Bloch et al., 1993), mais baixos e mais pesados do que os Suruí e os Zoró (Fleming-Moran et al., 1991) e mais baixos e mais leves que os índios americanos (Welty et al., 1995), do que a população brasileira urbana e rural não-indígena (Achutti \& Medeiros, 1985) e do que os Xavánte (Leite,
1998). Quando as médias de estatura são comparadas com as medidas de 43 grupos indígenas sul-americanos (Salzano \& Callegari-Jacques, 1988), observa-se que os valores Guaraní estão incluídos no intervalo de baixa a média estatura.

A média de IMC das mulheres Guaraní foi superior à dos homens, inversamente ao descrito por Bloch et al. (1993) nos Yanomámi. Na comparação com as médias de grupos indígenas americanos e brasileiros, as médias Guara- 
ní, de ambos os sexos, foram superiores às observadas nos Yanomámi (Bloch et al., 1993) e nos Suruí e nos Zoró (Fleming-Moran et al., 1991), que representam grupos com baixos níveis tensionais; foram inferiores às medidas observadas nos Xavánte (Leite, 1998), nos índios americanos (Welty et al., 1995; Greenlund et al., 1999), assim como na população brasileira não-indígena (Achutti \& Medeiros, 1985), todos considerados com elevadas prevalências de obesidade. Esse resultado sugere que os Guaraní apresentam-se em grau intermediário de obesidade entre os grupos comparados, fato que pode ser explicado pelas transformações observadas no padrão alimentar e de atividade física desenvolvida - em particular, pelas mulheres que, em geral, são responsáveis pelos afazeres domésticos e pelo artesanato.

As prevalências de sobrepeso nos Guaraní foram inferiores àquelas determinadas para a população brasileira (37\%: homens e 48\%: mulheres) (Schmidt \& Duncan, 1996). As mulheres Guaraní apresentaram maior prevalência de sobrepeso em relação aos homens, independentemente do grau, apesar das médias mais baixas de peso e estatura, tal qual observado nos índios americanos (Welty et al., 1995) e em populações brasileiras indígenas e não-indígenas (Leite, 1998; Sichieri et al., 1994).

O sobrepeso foi mais prevalente na faixa etária de 50 anos e mais, refletindo maior acúmulo de gordura corporal nas mulheres, sobretudo nas de maior idade, igualmente aos resultados encontrados por Pereira (1998) no Rio de Janeiro. Nos homens, a maior proporção de sobrepeso foi na faixa etária de 29 a 50 anos, quando apresentam a massa muscular mais desenvolvida, supostamente devido ao período em que os homens exercem atividades físicas de alta intensidade.

Paradoxalmente, o baixo peso também teve maior proporção no sexo feminino do que no masculino, estando igualmente mais representado na faixa etária de 50 anos e mais, como observado por Monteiro et al. (1995). Isso pode ser explicado pelas idades extremas - somente atingidas pelas mulheres -, que apresentam perda importante de massa muscular e menores índices de massa corporal. Retirando os indivíduos com idade superior a 80 anos da análise, a prevalência de baixo peso nas mulheres passou a existir somente na faixa etária de 15 a 29 anos.

Em contrapartida, o baixo peso no sexo masculino concentrou-se exclusivamente na faixa etária de 15 a 29 anos, a qual tem grande representatividade dos adolescentes, cuja massa muscular ainda está em desenvolvimento, cor- roborando a hipótese apresentada para a maior média de peso nos homens de idade intermediária. Supõe-se ainda que o baixo peso nos jovens possa representar um efeito coorte em decorrência de déficit nutricional das gerações mais novas e/ou também de exposição incipiente aos fatores determinantes da obesidade.

Apesar dos valores de IMC masculinos permanecerem, em sua maioria, dentro dos limites considerados adequados, cerca de $15 \%$ dos homens acima de 30 anos apresentaram RCQ elevada, sugerindo que, embora com peso conveniente, mostram concentração abdominal de gordura, o que possibilita maior capacidade preditiva às doenças crônicas (Pereira et al., 1999).

A quase totalidade das mulheres apresentou medidas de RCQ superiores ao valor tido como de risco para as doenças crônicas, embora os valores médios tenham sido maiores no grupo masculino, como observado também por Greenlund et al. (1999), Welty et al. (1995) e Schmidt et al. (1992). O comportamento dessa variável foi semelhante ao do IMC, com maior prevalência de alteração no sexo feminino, crescente com a idade, mostrando que as mulheres têm maior concentração abdominal de gordura que os homens, sobretudo as mais velhas.

A pressão arterial mostrou médias mais elevadas no sexo masculino, coincidentes com os valores do grupo Yanomámi (Bloch et al., 1993). Contudo, as médias de pressão encontradas foram intermediárias entre os resultados observados no grupo anteriormente citado (105,6 x $67,9 \mathrm{mmHg}$ ) e os resultados de estudos com populações indígenas americanas $(128,4$ x 79,5 mmHg) (Welty et al., 1995) e populações urbanas brasileiras $(122,4 \times 72,6 \mathrm{mmHg}$ ) (Klein et al., 1985). Quando resultados são comparados com valores médios de pressão arterial de 11 grupos indígenas sul-americanos, que apresentaram médias sistólicas e diastólicas masculinas variando, respectivamente, de 88,1 mmHg (Kalapálo e Kamayurá) a 117,9mmHg (Xavánte 2) e de 66,0mmHg (Kayapó) a 75,6 mmHg (Trio e Wayána), conclui-se que os níveis pressóricos do sexo masculino estão incluídos na escala de valores baixos a médios, correspondendo aproximadamente ao valor médio ponderado de todos os grupos $(109,1 \mathrm{x}$ $70,6 \mathrm{mmHg}$ ). No sexo feminino, as médias tensionais sistólica e diastólica dos 11 grupos variaram respectivamente de $78,7 \mathrm{mmHg}$ (Kalapálo e Kamayurá) a 117,4mmHg (Xavánte 2) e de 54,7mmHg (Kalapálo e Kamayurá) a 74,2 mmHg (Trio e Wayána), estando as médias das mulheres Guaraní situadas entre os valores médios e elevados. As médias sistólica e diastólica Guaraní foram superiores às médias pon- 
deradas de todos os grupos $(107,0 \times 68,5 \mathrm{mmHg})$ (Salzano \& Callegari-Jacques, 1988). Esses resultados demonstram que os Guaraní encontram-se em situação intermediária no que concerne aos níveis tensionais na comparação com grupos indígenas e não-indígenas.

Dos indivíduos avaliados, $4,8 \%$ foi classificado como hipertenso, diferentemente dos Yanomámi, onde ninguém foi classificado como tal. A prevalência foi inferior às encontradas em outros estudos realizados por Welty et al. (1995) e por Mendlein et al. (1997) em populações indígenas americanas $(26,8 \%$ e $17 \%)$, por Rego et al. (1990), na população de São Paulo (11,6\%), por Achutti \& Medeiros (11,3\%), no Rio Grande do Sul e por Lolio et al. (1993), na população de Araraquara (28,3\%). A prevalência de hipertensão no grupo estudado, pelo critério OMS, é inferior às encontradas para outras populações brasileiras não-indígenas igualmente classificadas, tais como as vistas no Rio Grande do Sul (13,7\% para homens e 10,0\% para mulheres), citadas por Rego et al. (1990) e por Klein et al. (1985), em Volta Redonda (10,1\%).

A prevalência de hipertensão arterial por sexo foi maior no grupo feminino, inversamente ao observado em todos os outros grupos submetidos à comparação, embora, na faixa etária de 15 a 29 anos, a prevalência tenha sido maior no grupo masculino, mostrando a reconhecida menor propensão das mulheres jovens à hipertensão. No grupo de 50 anos e mais, a prevalência se inverteu seja pela condição hormonal feminina ou pelo comportamento sedentário e obesidade observados. Nesse momento, notase um padrão comportamental da pressão arterial diferenciado do observado nas populações indígenas amazônicas, em geral. A pressão arterial aumenta com a idade em ambos os sexos, sendo significativo no grupo feminino, especificamente em relação à pressão sistólica. Da mesma forma, observa-se correlação positiva dos níveis tensionais com o IMC, peso, RCQ e com os lipídios em geral, sobretudo no sexo feminino. Como a RCQ apresentou correlação positiva com a idade e com os lipídios, pode-se supor que o acúmulo abdominal de gordura esteja intimamente correlacionado com o perfil tensional e lipídico adverso do grupo.

Várias condições poderiam estar contribuindo paralelamente para a situação encontrada, tais como a mudança do padrão alimentar, considerando que a suposta fase de exposição diferenciada dos sexos à ocidentalização do estilo de vida (Fleming-Moran et al., 1991) pudesse ter sido suplantada, acarretando a assimilação do consumo de carboidratos, gorduras e sal refinado ( INTERSALT, 1988; Leite, 1998;
Monteiro et al., 1995; Sichieri, 1998; Young, 1988) pelas mulheres. Outra explicação conseqüente da primeira seria a maior obesidade observada no grupo, sobretudo a central, aliada ao sedentarismo. Soma-se ainda maior acesso das mulheres aos serviços de atenção à saúde, permanentemente atuante na área, que poderia determinar relativa recuperação do estado físico feminino com maior resposta tensional (Fleming-Moran et al., 1991). Os resultados demonstram haver confluência de fatores de risco para as doenças crônicas não transmissíveis nesse grupo.

A média de colesterol das mulheres foi superior à dos homens, coincidente com os achados de Welty et al. (1995) no estudo de fatores de risco cardiovasculares em índios americanos (H: 190,2mg/dl e M: 192,6mg/dl) e oposto aos de Greenlund et al. (1999), em estudo sobre prevalência de variáveis correlatas à Síndrome de Resistência Insulínica, também em índios americanos (H: 214,0mg/dl e M: 210,5 $\mathrm{mg} / \mathrm{dl}$ ). A comparação das prevalências mostrou - inversamente aos estudos citados - valores discretamente mais elevados nas mulheres e inferiores aos dos indígenas americanos em geral. Esse fato aponta, apesar do suposto maior risco feminino, para um estado de má nutrição e/ou para um estágio ainda precoce na apresentação dos fatores de risco cardiovasculares nos Guaraní.

A média de colesterol-HDL posicionou-se dentro dos limites de referência para risco em ambos os sexos, sugerindo que a população estudada encontra-se propensa à aterogênese.

As prevalências de alteração de HDL observadas, superiores no grupo feminino e crescentes com a idade, confrontadas com os dados antropométricos, sugerem que a obesidade, a hipertrigliceridemia, o sedentarismo e o tabagismo (em menor grau) formam um conjunto de fatores concorrentes para os valores baixos de HDL encontrados e sua distribuição na população estudada.

Os valores de HDL correspondem à quinta parte do colesterol total. Os resultados podem ser ainda conseqüência dessa proporção, já que os valores de colesterol total foram baixos, levantando a necessidade de compreensão mais adequada do hábito alimentar atual, pois uma das causas de valores baixos dos lipídios é a já citada má nutrição, embora se observe certa tendência à obesidade.

A média e a prevalência de colesterol LDL foram igualmente superiores no grupo feminino, opostamente ao obtido nos estudos americanos, além de bastante inferiores aos valores observados nos mesmos. 
Os triglicerídios apresentaram média e prevalência maiores nas mulheres, constituindo uma das variáveis que mostrou a correlação mais forte com os índices antropométricos e com a pressão arterial, sobretudo nas mulheres. Esse achado é apoiado pelas citações de Pereira et al. (1999), Folson et al (1993) e Larsson et al. (1984) sobre a distribuição abdominal de gordura como indicador de risco cardiovascular (dislipidemias e HA), assim como pela afirmação de que o aumento de triglicerídios no plasma é indicativo de distúrbio metabólico.

Os resultados desse estudo permitem supor que os Guaraní-Mbyá do Estado do Rio de Janeiro apresentam-se em estágio intermediário entre as populações de estilo de vida ainda tradicional, há menos tempo contactadas, e aquelas que já se submeteram ao contato em tempos remotos ou mesmo populações urbanas, em relação à ocorrência de doenças crônicodegenerativas. Esse processo parece ser explicado por múltiplos fatores, sobressaindo a obesidade, sobretudo o acúmulo abdominal de gordura, que parece ser conseqüência das mudan- ças sócio-econômicas, comportamentais e do padrão alimentar, impostas pela escassez de recursos naturais para subsistência e por situação de vida adversa. As modificações do padrão de atividade física também demonstraram ser fator importante na determinação dos resultados observados no grupo, impondo mudanças no balanço alimentar e metabólico e integrando um círculo vicioso na fisiopatologia das doenças crônicas não transmissíveis.

Medidas de controle e acompanhamento dos casos existentes - prevenindo complicações, rastreamento e identificação permanente de novos casos - devem ser imediatamente adotadas. Acredita-se que, a longo prazo, medidas como um processo de auto-sustentação - com base na produção de fontes protéicas e de alimentos culturalmente aceitos, além de trabalhos educativos de orientação alimentar e combate ao sedentarismo - possam trazer bons resultados, uma vez que a manutenção dos hábitos tradicionais, em sua plenitude, não é possível, em decorrência da deterioração do ecossistema em que vivem.

\section{Referências}

ACHUTTI, A. \& MEDEIROS, A. M. B., 1985. Hipertensão arterial no Rio Grande do Sul. Boletim de Saúde, 12:6-54.

BLOCH, K. V.; COUTINHO, E. S. F.; LÔBO, M. E. C.; OLIVEIRA, J. E. P. \& MILECH, A., 1993. Pressão arterial, glicemia capilar e medidas antropométricas em uma população Yanomámi. Cadernos de Saúde Pública, 9:428-438.

CARVALHO, J. J. M.; BARUZZI, R. G.; HOWARD, P. F.; POULTER, N.; ALPERS, M. P.; FRANCO, L. J.; MARCOPITO, L. F; SPOONER, V. J.; DYER, A. R.; ELLIOTT, P.; STAMLER, J. \& STAMLER, R., 1989. Blood pressure in four remote populations in the INTERSALT Study. Hypertension, 14:238-246.

COIMBRA Jr., C. E. A. \& SANTOS, R. V., 1991. Avaliação do estado nutricional num contexto de mudança sócio-econômica: O grupo indígena Suruí do Estado de Rondônia, Brasil. Cadernos de Saúde Pública, 7:538-562.

DUSTAN, H. P., 1991. Hypertension and obesity. Primary Care, 18:495-507.

FLEMING-MORAN, M. \& COIMBRA Jr., C. E. A., 1990. Blood pressure studies among amazonian native populations: A review from an epidemiological perspective. Social Science and Medicine, 31:593601 .
FLEMING-MORAN, M.; SANTOS, R. V. \& COIMBRA Jr., C. E. A., 1991. Blood pressure levels of the Suruí and Zoró Indians of the Brazilian Amazon: Group- and sex-specific effects resulting from body composition, health status, and age. $\mathrm{Hu}$ man Biology, 63:835-861.

FOLSON, A. R.; KAYE, S. A.; SELLERS, T. A.; HONG, C.P.; CERHAN, J. R.; POTTER, J. D. \& PRINEAS, R. J., 1993. Body fat distribution and 5-year risk of death in older women. JAMA, 269:483-487.

FUCHS, F. D., 1996. Hipertensão arterial sistêmica. In: Medicina Ambulatorial: Condutas Clínicas em Atenção Primária (B. B. Duncan, M. I. Schimidt \& E. R. J. Giugliani, org.), pp. 433-443, Porto Alegre: Editora Artes Médicas Sul.

GREENLUND, K. J.; VALDEZ, R.; CASPER, M. L.; RITH-NAJARIAN, S. \& CROFT, J. B., 1999. Prevalence and correlates of the Insulin Resistance Syndrome Among Native Americans - The InterTribal Heart Project. Diabetes Care, 22:441-447.

HERN, W. M., 1991. Health and demography of Native Amazonians: Historical perspective and current status. Cadernos de Saúde Pública, 7:451-480.

INTERSALT (International Study of Electrolyte Excretion and Blood Pressure), 1988. Intersalt: An International Study of Electrolyte Excretion and 
Blood Pressure. Results for 24 hour urinary sodium and potassium excretion. BMJ, 297:319-328.

JNC (Joint National Committe), 1988. The 1988 Report of the JNC on detection, evaluation, and treatment of high blood pressure. Archives of Internal Medicine, 148:1023-1038.

KLEIN, C. H.; ARAÚJO, J. W. G. \& LEAL, M. C., 1985. Inquérito epidemiológico sobre hipertensão arterial em Volta Redonda - RJ. Cadernos de Saúde Pública, 1:58-70.

LARSSON, B.; SVÄRDSUDD, K.; WELIN, L.; WILHELMSEN, L.; BJÖRNTORP, P. \& TIBBLIN, G. 1984. Abdominal adipose tissue distribution, obesity, and risk of cardiovascular disease and death: 13 year follow up of participants in the study of men born in 1913. BMJ, 288:1401-1404.

LEITE, M. S., 1998. Avaliação do Estado Nutricional da População Xavánte de São José, Terra Indígena Sangradouro - Volta Grande, Mato Grosso. Dissertação de Mestrado, Rio de Janeiro: Escola Nacional de Saúde Pública, Fundação Oswaldo Cruz.

LITAIFF, A., 1996. As Divinas Palavras: Identidade Étnica dos Guaraní-Mbyá. Florianópolis: Editora da Universidade Federal de Santa Catarina.

LOLIO, C. A.; PEREIRA, J. C. R.; LOTUFO, P. A.; SOUZA, J. M. P., 1993. Hipertensão arterial e possíveis fatores de risco. Revista de Saúde Pública, 27:357-362.

MENDLEIN, J. M.; FREEDMAN, D. S.; PETER, D. G.; ALLEN, B.; PERCY, C. A.; BALLEW, C.; MOKDAD, A. H. \& WHITE, L. L., 1997. Risk factors for coronary heart disease among Navajo Indians: Findings from the Navajo Health and Nutrition Survey. Journal of Nutrition, 127: 2099S-2105S.

MONTEIRO, C. A.; MONDINI, C. A.; SOUZA, A. L. M. \& POPKIN, B. M., 1995. Da desnutrição para a obesidade: A transição nutricional no Brasil. In: Velhos e Novos Males da Saúde no Brasil - A Evolução do País e de Suas Doenças (C. A. Monteiro, org.), pp. 247-255, São Paulo: Editora Hucitec/Núcleo de Pesquisas Epidemiológicas em Nutrição e Saúde, Universidade de São Paulo.

NCEP (National Cholesterol Education Program), 1993. Summary of the second report of The National Cholesterol Education Program (NCEP) Expert Panel on Detection, Evolution, and Treatment of High Blood Cholesterol in Adults (Adult Treatment Panel II). JAMA, 269:3015-3023.

PERCY, C.; FREEDMAN, D. S.; GILBERT, T. J.; WHITE, L. \& BALLEW, C., 1997. Prevalence of hypertension among Navajo Indians: Findings from the Navajo Health and Nutrition Survey. Journal of Nutrition, 127:2114S-2119S.

PEREIRA, R. A., 1998. Avaliação antropométrica do estado nutricional. In: Epidemiologia da Obesidade (R. Sichieri, org.), pp. 43-64, Rio de Janeiro: EdUERJ.

PEREIRA, R. A.; SICHIERI, R. \& MARTINS, V. M. R., 1999. Razão cintura/quadril como preditor de hipertensão arterial. Cadernos de Saúde Pública, 15:333-344

REGO, A. R.; BERARDO, F. A. N.; RODRIGUES, S. S. R.; OLIVEIRA, Z. M. A.; OLIVEIRA, M. B.; VASCONCELLOS, C.; AVENTURATO, L. V. O.; MONCAU, J. E. C. \& RAMOS, L. R., 1990. Fatores de risco para doenças crônicas não-transmissíveis: Inquérito domiciliar no município de São Paulo, SP (Brasil). Metodologia e resultados preliminares. Revista de Saúde Pública, 24:277-285.

RHOADES, E.; HAMMOND, J.; WELTY, T. K.; HANDLER, A. O. \& AMLER, R. W., 1987. The indian burden of illness and future health interventions. Public Health Reports, 102:361-368.

SICHIERI, R., 1998. Epidemiologia da Obesidade. Rio de Janeiro: EdUERJ.

SICHIERI, R.; COITINHO, D. C.; LEÃO, M. M.; RECINE, E. \& EVERHART, J. E., 1994. High temporal, geographic, and income variation in body mass index among adults in Brazil. American Journal of Public Health, 84:793-797.

SALZANO, F. M. \& CALLEGARI-JACQUES, S. M., 1988. South American Indians: A Case Study in Evolution. Oxford: Clarendon Press.

SANTOS, R. V. \& COIMBRA Jr., C. E. A., 1994. Contato, mudanças sócio-econômicas e a bioantropologia dos Tupi-Mondé da Amazônia Brasileira. In: Saúde e Povos Indígenas (R. V. Santos \& C. E. A. Coimbra Jr., org.), pp. 189-211, Rio de Janeiro: Editora Fiocruz.

SCHMIDT, M. I. \& DUNCAN, B. B., 1996. Obesidade. In: Medicina Ambulatorial: Condutas Clínicas em Atenção Primária (B. B. Duncan, M. I. Schmidt \& E. R. J. Giugliani, org.), pp. 280-286, Porto Alegre: Editora Artes Médicas Sul.

SCHMIDT, M. I.; DUNCAN, B. B.; CANANI, L. H.; KAROHL, C. \& CHAMBLESS, L., 1992. Association of waist-hip ratio with Diabetes mellitus. Diabetes Care, 15:912-914.

WELTY, T. K.; LEE, E. T.; YEH, J.; COWAN, L. D.; GO, O.; FABSITZ, R. R.; LE, N.-A.; OOPIK, A. J.; ROBBINS, D. C. \& HOWARD, B. V., 1995. Cardiovascular disease risk factors among American Indians - The Strong Heart Study. American Journal of Epidemiology, 142:269-287.

WHO (World Health Organization), 1978. Arterial Hipertension. Report of a WHO Expert Committee. Technical Report Series 628. Geneva: WHO.

WHO (World Health Organization), 1995. Physical Status: The Use and Interpretation of Anthropometry - Report of a WHO Expert Commitee. WHO Technical Report Series 854. Geneva: WHO.

YOUNG, T. K., 1988. Are Subartic Indians undergoing the Epidemiologic Transition? Social Science and Medicine, 26:659-671. 